\title{
Qualidade de vida em crianças portadoras do Vírus da Imunodeficiência Humana (HIV)
}

\section{Quality of life in children with the Human Immunodeficiency Virus (HIV)}

\author{
Thuanny Silva de Macêdo ${ }^{*}$ (D), Maria José Rodrigues² (D, \\ Maria das Graças Félix de Figueiredo ${ }^{3}$ (D) , Valdeci Elias dos Santos Júnior ${ }^{4}$ (D) \\ 'Programa de Pós-graduação em Odontologia, Universidade Federal de Pernambuco (UFPE) - Recife (PE), Brasil. \\ ${ }^{2}$ Faculdade de Odontologia de Pernambuco, Universidade de Pernambuco (UPE) - Camaragibe (PE), Brasil. \\ ${ }^{3}$ Instituto de Medicina Integral Professor Fernando Figueira (IMIP) - Recife (PE), Brasil. \\ ${ }^{4}$ Universidade de Pernambuco (UPE) - Recife (PE), Brasil.
}

Como citar: Macêdo TS, Rodrigues MJ, Figueiredo MGF, Santos Júnior VE. Qualidade de vida em crianças portadoras do Vírus da Imunodeficiência Humana (HIV). Cad Saúde Colet, 2020;28(2):223-230. https://doi.org/10.1590/1414$462 \times 202028020233$

\section{Resumo}

Introdução: O estudo da qualidade de vida de crianças portadoras de doenças crônicas, como o HIV, é essencial, visando um cuidado integral e holístico para os portadores da doença. Objetivo: Avaliar a qualidade de vida e os fatores sociodemográficos associados de crianças portadoras do HIV. Método: Estudo descritivo transversal, amostra de 78 crianças de ambos os sexos, faixa etária de 3 a 13 anos, atendidas em serviço de referência da cidade do Recife-PE. Foram utilizados o questionário Critério de Classificação Econômica Brasil, para caracterização socioeconômica, e a Escala de Qualidade de Vida da Criança (AUQEI). Para avaliar associação entre as variáveis, foram utilizados o teste Qui-quadrado de Pearson e o teste Exato de Fischer. A margem de erro utilizada foi de $5 \%$. Resultados: No tocante à qualidade de vida, ficar doente, brincar sozinho e a presença de bullying na escola apresentaram baixos índices de satisfação. Já itens como férias, aniversário, estar à mesa com a família e assistir televisão apresentaram altos níveis de satisfação. Conclusão: As crianças desta pesquisa demonstraram uma qualidade de vida influenciada pelos aspectos negativos presentes em suas vidas. Desta forma, é necessária uma equipe multidisciplinar para o tratamento e prevenção de comorbidades, inclusive as psicossociais.

Palavras-chave: qualidade de vida; crianças; HIV/AIDS.

\begin{abstract}
Background: Studying the quality of life of children with chronic diseases, such as HIV, is essential, aiming at comprehensive and holistic care for people with the disease. Objective: To assess the quality of life and sociodemographic associated factors in children with HIV. Method: A descriptive cross-sectional study, with a sample of 78 children of both genders, aged between 3 and 13 years old, attending a reference service in the city of Recife-PE. Two questionnaires were used to collect data, the Brazilian Economic Classification Criteria questionnaire, to accomplish the socioeconomic characteristics, and the Quality of Life Scale for Children (AUQEI). Pearson's Chi-square test and Fisher's exact test evaluated the association between the variables. The margin of error was $5 \%$. Results: The quality of life, being sick, playing alone and the presence of bullying in school had low satisfaction rates. On the other hand, items such as vacations, birthday, being at the table with the family, and watching television showed high levels of satisfaction. Conclusions: The children in this research demonstrated a quality of life influenced by the negative aspects present in their lives. Thus, a multidisciplinary team is needed for the treatment and prevention of comorbidities, including psychosocial ones.
\end{abstract}

Keywords: quality of life; children; HIV/AIDS.

Este é um artigo publicado em acesso aberto (Open Access) sob a licença Creative Commons Attribution, que permite uso, distribuição e reprodução em qualquer meio, sem restrições desde que o trabalho original seja corretamente citado.
Trabalho realizado no Instituto de Medicina Integral Professor Fernando Figueira (IMIP), Recife (PE), Brasil.

Correspondência: Thuanny Silva de Macêdo. E-mail: thuannymacedo16@gmail.com

Fonte de financiamento: nenhuma.

Conflito de interesses: nada que declarar.

Recebido em: Jul. 10, 2017. Aprovado: Jul. 23, 2019 


\section{INTRODUÇÃO}

A epidemia da infecção pelo vírus da Imunodeficiência Humana (HIV) e a Síndrome da Imunodeficiência Humana (AIDS), desde a sua descoberta em 1981, vem se mostrando como um fenômeno global, contínuo, instável e dinâmico, e ainda é considerada um dos maiores problemas de saúde pública no Brasil e no mundo'. É um desafio em diversos aspectos, sobretudo, pela ausência de um tratamento efetivo que conduza à cura, além das barreiras sociais e econômicas que interferem na adesão ao regime terapêutico ${ }^{2}$.

O HIV/AIDS é um desafio emergente de saúde pública, pautado na busca da saúde integral e da qualidade de vida ${ }^{3}$. O vírus HIV e, em consequência, a AIDS estão ganhando proporções cada vez maiores desde sua descoberta, pois disseminaram-se em toda população, sem fazer distinção de idade, raça ou sexo 4 .

A ocorrência de mulheres infectadas pelo HIV resultou no nascimento de crianças expostas ou infectadas pelo vírus ${ }^{5}$. De acordo com diretrizes nacionais, a definição de casos de AIDS em crianças é todo indivíduo com menos de 13 anos de idade que apresentar evidência laboratorial de infecção pelo HIV e alguma evidência de imunodeficiência6.

Os riscos de infecção enfrentados pelo feto de uma mãe vivendo com HIV são minimizados quando os diagnósticos disponíveis e medicamentos antirretrovirais são utilizados no tempo correto. Globalmente, o acesso a medicamentos contra o HIV para prevenir a transmissão do vírus de mãe para filho aumentou para 77\% em 2015 (acima dos 50\% registrados em 2010). Como resultado, as novas infecções por HIV entre crianças diminuíram 51\% desde $2010^{7,8}$.

Diante da transmissão vertical do HIV, permanecem ações fundamentais, que incluem educação e uso de preservativos durante a relação sexual. Estas, juntamente com a profilaxia da transmissão vertical, não são capazes de eliminar a infecção pelo HIV na população pediátrica, mas são potencialmente viáveis dentro do sistema público de saúde 9 .

O Programa Conjunto das Nações Unidas sobre HIV/AIDS (UNAIDS) divulgou que no ano de 2015 existiam cerca de 36,7 milhões de pessoas no mundo vivendo com o HIV, e destas, 1,8 milhão possuíam menos que 15 anos de idade. Apenas no ano de 2015, 150 mil novas crianças foram infectadas pelo vírus em todo o mundo, e neste mesmo ano ocorreram $110 \mathrm{mil}$ mortes relacionadas à AIDS em menores de 15 anos $^{10,11}$. Entre os anos de 1980 e 2016 foram notificados 16.381 casos de AIDS em menores de 13 anos de idades no Brasil. Quanto à categoria de exposição entre os indivíduos menores de 13 anos, a quase totalidade dos casos $(93,0 \%)$ teve como via de infecção a transmissão vertical ${ }^{12}$.

A Organização Mundial de Saúde (OMS) define qualidade de vida como a percepção que o indivíduo tem de sua posição na vida, considerando o contexto da cultura e sistemas de valores nos quais ele está inserido, e em relação a seus objetivos, suas expectativas, padrões e preocupações ${ }^{13,14}$.

A compreensão da qualidade de vida dos portadores de HIV/AIDS é fundamental, considerando a evolução crônica da infecção, possibilidade de tratamento, maior sobrevida e convívio com uma doença estigmatizante, transmissível e incurável até o momento, com inúmeras consequências biopsicossociais que repercutem na qualidade de vida ${ }^{15}$. Essa realidade indica a necessidade de se cuidar da criança e da sua família de forma holística, considerando não somente aspectos biológicos e de tratamento da doença, mas, sobretudo, o entorno social e ambiental no qual estão inseridas ${ }^{16,17}$.

Essas crianças ainda estão iniciando seu processo de interação social e conhecimento do mundo onde vivem, e acabam tendo profundas dificuldades para se inserir em uma sociedade incapaz de ser acolhedora e de conviver com as diferenças. Embora as crianças possam sentir, ainda não sabem fazer julgamentos e entender a situação à qual estão expostas ${ }^{5}$.

Vê-se necessária então a avaliação da QV infantil, considerando a criança como ator principal, preconizando, assim, sua interpretação sobre o meio, possibilitando diagnósticos de fatores que contribuem para a insatisfação ou a perturbação do seu processo de desenvolvimento, a fim de melhorar a qualidade de vida desses núcleos familiares e o atendimento a essas crianças e esses adolescentes, suprindo todas as suas necessidades ${ }^{18}{ }^{19}$. Diante disto, este estudo objetivou a avaliação da qualidade de vida e dos fatores sociodemográficos associados de crianças portadoras do HIV. 


\section{MÉTODO}

Trata-se de um estudo descritivo transversal desenvolvido em um centro de referência nacional para o HIV infantil, localizado no município de Recife-PE, no período de julho de 2014. Participaram do estudo crianças entre 3 e 13 anos de idade, portadoras do vírus HIV e que se encontravam em atendimento no centro no período acima referenciado. Seriam perdidas as crianças que não aceitassem responder ao questionário sobre Qualidade de Vida (QV) ou aquelas cujos responsáveis não aceitassem responder ao questionário sobre os fatores socioeconômicos, porém não houve recusas durante o desenvolvimento do estudo.

A amostra foi selecionada por livre demanda, sendo assim, foi composta pelo número de crianças que compareceram para o atendimento durante o período de coleta de dados. Nesse período, foram abordados 118 pacientes, dentre estes, 40 foram excluídos por não se enquadrarem nos critérios de inclusão (crianças menores de 3 anos de idade), resultando assim em uma amostra de 78 pacientes.

Para coleta dos dados utilizaram-se dois instrumentos. Para avaliar a QV, utilizou-se o questionário Escala de Qualidade de Vida da Criança (Autoquestionnaire Qualité de Vie Enfant Imagé - AUQEI), desenvolvido por Manificat e Dazord ${ }^{20} \mathrm{e}$ traduzido e validado para o português por Assumpção et al. ${ }^{21}$. O questionário também passou por um processo de validação em crianças infectadas pelo HIV, realizado no Brasil por Ferreira et al. ${ }^{22}$.

Esse instrumento é baseado no ponto de vista da satisfação da criança, visualizada a partir de quatro figuras que são associadas a diversos domínios da vida, através de 26 questões que exploram relações familiares, sociais, atividades, saúde, funções corporais e separação (Quadro 1).

Quadro 1. Domínios estudados na Escala de Avaliação de Qualidade de Vida da Criança

\begin{tabular}{l|l|c|}
\multicolumn{1}{c|}{ Domínios } & \multicolumn{1}{|c}{-} & Questões \\
\hline Autonomia & $\begin{array}{l}\text { Relativo à independência, relações com companheiros e } \\
\text { avaliações }\end{array}$ & $12,15,17,18,19,24$ \\
\hline Lazer & Relativo a férias, aniversário, relações com avós & $7,9,11,21,25,26$ \\
\hline Funções & Relativo à atividade na escola, a refeições, deitar, ida ao médico & $1,2,4,5,8,14,20$ \\
\hline Família & $\begin{array}{l}\text { Relativo à opinião quanto às figuras parentais e delas quanto a } \\
\text { si mesmo }\end{array}$ & $3,6,10,13,16,22,23$ \\
\hline
\end{tabular}

Cada resposta recebe uma pontuação de 0 a 3 , sendo o primeiro correspondente ao estado muito infeliz e o último ao estado muito feliz. O somatório dos escores de todas as questões representa o total individual, podendo este valor variar de 0 a 78. De acordo com o estudo de validação do questionário, a pontuação de corte utilizada foi de 48 , sendo a qualidade de vida considerada prejudicada nos casos em que a pontuação for inferior a esta nota ou satisfatória nas crianças com escores iguais ou superiores a 48 pontos $^{21}$.

O questionário Critério de Classificação Econômica Brasil (CCEB) fez a caracterização socioeconômica das famílias nas quais as crianças viviam e foi aplicado aos pais e/ou cuidadores. Este questionário consegue avaliar o poder de compra das famílias e o grau de escolaridade (para crianças que apresentavam mais de um responsável, o dado registrado foi o do responsável com maior escolaridade), classificando-os então, de acordo com os pontos obtidos, em classes econômicas: A1 (42 a 46 pontos); A2 (35 a 41 pontos); B1 (29 a 34 pontos); B2 ( 23 a 28 pontos); C1 (18 a 22 pontos); C2 ( 14 a 17 pontos); $D$ ( 8 a 13 pontos) e E ( 0 a 7 pontos).

Os participantes foram entrevistados em seus dias de consulta médica mensal. Inicialmente havia a explicação do presente estudo aos responsáveis, e para aqueles que concordassem em participar solicitava-se que assinassem o Termo de Consentimento Livre e Esclarecido, tanto consentindo na sua participação como na da criança/adolescente, e o Termo de Assentimento (assinado por crianças com idades entre 6 e 13 anos). 
A partir daí, em uma sala separada, fez-se a entrevista para a coleta de dados. Primeiramente, os responsáveis respondiam ao Questionário CCEB, e em seguida era aplicado às crianças o questionário $A \cup Q E I$. Nas crianças mais novas, que não apresentavam total capacidade de leitura, o questionário era aplicado na forma de entrevista.

Os resultados foram analisados descritivamente através de percentuais e de medidas estatísticas: média e desvio-padrão. Para avaliar a associação entre as variáveis, foram utilizados o teste Qui-quadrado de Pearson e o teste Exato de Fischer. A margem de erro utilizada na decisão dos testes estatísticos foi de 5\%. Os dados foram digitados em planilha EXCEL, e o programa utilizado para obtenção dos cálculos estatísticos foi o Statistical Package for Social Sciences (SPSS) na versão 21.

O estudo obedeceu à Resolução 466/12 do Conselho Nacional de Saúde, que normatiza a pesquisa envolvendo seres humanos. Assim, foi aprovado pelo Comitê de Ética e Pesquisa (CEP) do Instituto de Medicina Integral Professor Fernando Figueira (IMIP) sob o parecer n $41176-14$.

\section{RESULTADOS}

A idade das crianças pesquisadas variou de 3 a 13 anos, apresentando média de 7,47 anos e um desvio-padrão de 4,168 anos, e dos 78 pesquisados, 38 (48,7\%) eram do sexo masculino e $40(51,3 \%)$ do feminino. Foi possível observar que a maioria $(56,6 \%)$ dos responsáveis entrevistados possui o primeiro grau completo e apenas um (1,3\%) dos participantes possui o ensino superior completo. A maioria se enquadrou na classe C2 (41\%) e possuindo uma renda familiar média de 1.277 reais.

Em relação à qualidade de vida, os escores do questionário AUQEI variaram de 30 a 65, sendo a média de 46,76 . A maior parte $(n=42,53,8 \%)$ dos participantes foram enquadrados no grupo da qualidade de vida prejudicada, o que significa que a maioria dos escores obtidos foram menores do que 48 . E 36 participantes $(46,2 \%)$ foram enquadrados no grupo da qualidade de vida não prejudicada.

Avaliando os resultados referentes aos domínios de QV propostos pelo questionário AUQEI, o domínio do Lazer apresentou os maiores índices de satisfação, enquanto o domínio das Funções apresentou os menores índices (Tabela 1).

Tabela 1. Distribuição das crianças pesquisadas quanto aos domínios e às respostas do questionário AUQEI

\begin{tabular}{|c|c|c|c|c|c|c|c|c|}
\hline \multirow{2}{*}{ Domínios de QV } & \multicolumn{2}{|c|}{$\begin{array}{l}\text { Muito } \\
\text { infeliz }\end{array}$} & \multicolumn{2}{|c|}{ Infeliz } & \multicolumn{2}{|c|}{ Feliz } & \multicolumn{2}{|c|}{ Muito Feliz } \\
\hline & $\mathbf{N}$ & $\%$ & $\mathbf{N}$ & $\%$ & $\mathbf{N}$ & $\%$ & $\mathbf{N}$ & $\%$ \\
\hline \multicolumn{9}{|l|}{ Funções: } \\
\hline À mesa com a família & - & - & 7 & 9 & 57 & 73,1 & 14 & 17,9 \\
\hline Ao deitar & - & - & 7 & 9 & 59 & 75,6 & 12 & 15,4 \\
\hline Ao dormir & 1 & 1,3 & 6 & 7,7 & 59 & 75,6 & 12 & 15,4 \\
\hline Na sala de aula & 1 & 1,3 & 9 & 11,5 & 49 & 62,8 & 19 & 24,4 \\
\hline Na consulta médica & 16 & 20,5 & 31 & 39,7 & 30 & 38,5 & 1 & 1,3 \\
\hline Ao ficar internado no hospital & 53 & 67,9 & 19 & 24,4 & 6 & 7,7 & - & - \\
\hline Ao tomar os remédios & 28 & 35,9 & 30 & 38,5 & 20 & 25,6 & - & - \\
\hline \multicolumn{9}{|l|}{ Família: } \\
\hline Ao brincar com irmãos & - & - & 10 & 12,8 & 36 & 46,2 & 32 & 41,0 \\
\hline Ao ver uma fotografia sua & 2 & 2,6 & 3 & 3,8 & 41 & 52,6 & 32 & 41,0 \\
\hline Ao pensar no pai & 16 & 20,5 & 18 & 23,1 & 27 & 34,6 & 17 & 21,8 \\
\hline
\end{tabular}


Tabela 1. Continuação...

\begin{tabular}{|c|c|c|c|c|c|c|c|c|}
\hline \multirow{2}{*}{ Domínios de QV } & \multicolumn{2}{|c|}{$\begin{array}{l}\text { Muito } \\
\text { infeliz }\end{array}$} & \multicolumn{2}{|c|}{ Infeliz } & \multicolumn{2}{|c|}{ Feliz } & \multicolumn{2}{|c|}{ Muito Feliz } \\
\hline & $\mathbf{N}$ & $\%$ & $\mathbf{N}$ & $\%$ & $\mathbf{N}$ & $\%$ & $\mathbf{N}$ & $\%$ \\
\hline Ao pensar na mãe & 11 & 14,1 & 10 & 12,8 & 28 & 35,9 & 29 & 37,2 \\
\hline Quando o Pai/Mãe fala de você & 15 & 19,2 & 35 & 44,9 & 28 & 35,9 & - & - \\
\hline Ao pensar em quando tiver crescido & - & - & 1 & 1,3 & 41 & 52,6 & 36 & 46,2 \\
\hline Quando está longe da família & 16 & 20,5 & 54 & 69,2 & 7 & 9,0 & 1 & 1,3 \\
\hline \multicolumn{9}{|l|}{ Lazer: } \\
\hline Durante o recreio escolar & - & - & 8 & 10,3 & 41 & 52,6 & 29 & 37,2 \\
\hline Ao praticar um esporte & 1 & 1,3 & 5 & 6,4 & 53 & 67,9 & 19 & 24,4 \\
\hline No dia do aniversário & - & - & 4 & 5,1 & 45 & 57,7 & 29 & 37,2 \\
\hline Durante as férias & - & - & 1 & 1,3 & 28 & 35,9 & 49 & 62,8 \\
\hline Ao pensar nos avós & 5 & 6,4 & 3 & 3,8 & 45 & 57,7 & 25 & 32,1 \\
\hline Ao assistir televisão & 1 & 1,3 & 3 & 3,8 & 44 & 56,4 & 30 & 38,5 \\
\hline \multicolumn{9}{|l|}{ Autonomia: } \\
\hline Ao fazer a lição de casa & - & - & 24 & 30,8 & 50 & 64,1 & 4 & 5,1 \\
\hline Brincar sozinho & 21 & 26,9 & 19 & 24,4 & 18 & 23,1 & - & - \\
\hline Ao dormir fora de casa & 3 & 3,8 & 10 & 12,8 & 54 & 69,2 & 11 & 14,1 \\
\hline Ao mostrar algo que sabe fazer & 2 & 2,6 & 11 & 14,1 & 42 & 53,8 & 23 & 29,5 \\
\hline Quando amigos falam de você & 29 & 37,2 & 39 & 50,0 & 10 & 12,8 & - & - \\
\hline Ao receber as notas da escola & - & - & 21 & 26,9 & 41 & 52,6 & 16 & 20,5 \\
\hline
\end{tabular}

Quanto à distribuição dos pesquisados, no que diz respeito às respostas dos itens do questionário $A \cup Q E I$, as crianças apresentaram índices elevados de satisfação nas questões referentes ao pensar em quando tiverem crescido, durante as férias, ao assistir televisão, no dia do aniversário, ao verem uma fotografia sua, ao praticar esportes e ao estar à mesa com a família. De maneira inversa, os itens concernentes às situações de quando vai a uma consulta médica, ao ficar internado em um hospital, ao brincar sozinho, ao tomar os remédios, ao ficar longe da família e quando os amigos falam sobre a criança, obtiveram baixos índices de satisfação (Tabela 1).

Foi possível perceber que o sexo feminino obteve maior porcentagem (60\%) de participantes com a QV prejudicada do que os participantes do sexo masculino, que apresentaram $40 \%$ dos participantes com a QV prejudicada. Apesar dessa diferença, percebeu-se, através do teste Qui-quadrado de Pearson, que ela não foi estatisticamente significativa $(p=0,236)$.

Quando avaliada a associação entre escolaridade e qualidade de vida, e entre classe social e qualidade de vida, foi possível observar, por meio do Teste Exato de Fisher, que não houve diferença significativa do ponto de vista estatístico ( $p=0,701$ e $p=0,380$, respectivamente).

\section{DISCUSSÃO}

No tocante a trabalhos realizados com pacientes com doenças crônicas, como o HIV, a amostra pode parecer pequena, entretanto, estudos que avaliam a qualidade de vida em crianças com comprometimento sistêmico, em geral, têm uma amostra menor pela dificuldade em se conseguir participantes dentro de uma determinada faixa etária e com a patologia que se quer avaliar ${ }^{19,23}$. 
A idade dos pesquisados deste estudo variou entre 3 e 13 anos, apresentando uma média de 7,47 anos. Essa média de idade foi mais baixa do que a observada em outros estudos ${ }^{23-26}$.

Entre os 78 pesquisados, 40 eram do sexo feminino e 38, do masculino. Dados esses que são semelhantes aos obtidos por outros estudos que avaliaram crianças portadores de HIV e também encontraram uma maior prevalência do sexo feminino ${ }^{19,23-26}$.

Neste estudo, percebeu-se que o sexo feminino obteve maior porcentagem de participantes com a QV prejudicada, porém a diferença não foi estatisticamente significante. Já um estudo realizado com a finalidade de averiguar a qualidade de vida de pré-escolares, ao analisar o escore geral da percepção de QV nos participantes do estudo, foi verificado que os meninos apresentaram nível de satisfação maior do que as meninas ${ }^{18}$. Porém, indo de acordo com os dados do presente estudo, um trabalho que avaliou a QV de crianças infectadas pelo HIV/AIDS constatou que não houve diferença estatisticamente significativa entre os sexos, em relação aos escores no AUQEl'.

A avaliação dos dados referentes à escolaridade e à classe econômica dos pais e responsáveis mostrou dados semelhantes aos observados na literatura, trazendo a classe $C$ como a mais frequente nas famílias de crianças infectadas pelo HIV, seguida das classes $\mathrm{D}, \mathrm{B} 2 \mathrm{e} \mathrm{B} 1^{19}$. A literatura também mostra altos índices de dificuldades socioeconômicas que podem influenciar negativamente nas condições de saúde: a maioria vive em situação de pobreza, baixa escolaridade e desemprego, fatores que podem influenciar negativamente o crescimento e o desenvolvimento dessas crianças ${ }^{2,5}$.

Torna-se interessante pontuar que a assistência às pessoas infectadas pelo HIV requer de seu cuidador maior conhecimento e preparo. A baixa condição socioeconômica, que implica o grau de instrução do mantenedor da família, pode gerar problemas na adesão às terapias, bem como afetar a capacidade de enfrentamento da doença ${ }^{27}$.

Os índices de QV do presente estudo foram inferiores aos encontrados em outras pesquisas. Um estudo realizado com a finalidade de validar o questionário AUQEI fez a sua aplicação em 353 crianças, com idade entre 4 e 12 anos, e obteve um escore médio de 52,1 pontos ${ }^{21}$.

Em uma pesquisa realizada com 59 crianças de 6 a 12 anos infectadas pelo HIV, o escore médio do AUQEI foi de 50,29 pontos, e os resultados mostraram que a maioria (74,6\%) das crianças obteve um escore maior que 48 , o que é considerado uma qualidade de vida aceitável ${ }^{23}$. Em outro estudo, que objetivou identificar, descrever e analisar os indicadores de QV de crianças e adolescentes convivendo com HIV/AIDS, foi aplicado o questionário AUQEI em 30 participantes, e os resultados mostraram escores médios de 53,2, apontando boa qualidade de vida entre os participantes ${ }^{25}$.

O domínio referente ao Lazer apresentou os melhores índices de satisfação entre as crianças participantes do estudo, dentro deste item estando incluídas questões referentes a férias, aniversário e relação com os avós. Esse achado, que corrobora dados encontrados na literatura ${ }^{22}$, ilustra o impacto que atividades de lazer possuem na infância, evidenciando a importância do componente lúdico no dia a dia dessas crianças, independentemente de sua condição de saúde.

O presente estudo encontrou o domínio das Funções como o que apresentou os piores índices de satisfação. Em contrapartida, um estudo realizado com o objetivo de avaliar a QV de pré-escolares saudáveis através do questionário AUQUE| ${ }^{18}$ encontrou o domínio da Autonomia como o mais prejudicado. Acredita-se que esta diferença está relacionada com o fato de que, quando se trata de crianças portadoras de alguma patologia, atividades referentes aos cuidados com a saúde se tornam mais indesejadas, e este fato torna-se ainda mais relevante em se tratando de crianças infectadas pelo HIV, já que elas apresentam episódios frequentes de internação e fazem uso constante de medicamentos ${ }^{23}$, itens esses que estão contidos no domínio das funções.

O perfil de respostas das crianças apresentou índices elevados de satisfação nas questões referentes ao pensar em quando tiverem crescido, durante as férias, ao assistir televisão, no dia do aniversário, ao verem uma fotografia sua, ao praticar esportes e ao estar à mesa com a família. De maneira inversa, os itens concernentes às situações de quando vai a uma consulta médica, ao ficar internado em um hospital, ao brincar sozinho, ao tomar os remédios, ao ficar longe 
da família e quando os amigos falam sobre a criança, obtiveram baixos índices de satisfação. Esses dados corroboram os resultados obtidos em outras pesquisas ${ }^{21,23,25}$.

Apesar de não representarem a maioria, $43,6 \%$ dos participantes relataram sentimentos negativos (infeliz ou muito infeliz) quando questionados sobre o seu pai, e $26,9 \%$ relataram estes mesmos sentimentos quando questionados sobre a sua mãe. Porém, há que se destacar que o cotidiano de uma criança infectada pelo HIV nem sempre vem acompanhado de uma figura paterna ou materna. Muitas dos pais morrem, outros estão incapacitados devido às condições físicas e psicológicas. Em várias situações, a criança é institucionalizada por falta de um cuidador familiar, e um tutor assume o papel, tornando-se a maior influência para essas crianças $^{28}$.

Em uma pesquisa realizada com o objetivo de identificar aspectos das interações sociais de crianças portadoras de HIV pela ótica de seus cuidadores, os resultados mostraram que as interações sociais das crianças revelaram-se prejudicadas. Medo, exclusão e preconceito permeiam a vida dos cuidadores, pelo receio de as crianças serem discriminadas pela sociedade ${ }^{5}$.

A criança portadora do HIV/AIDS necessita então, além do tratamento médico, do acompanhamento de uma equipe multidisciplinar, em razão de apresentar uma morbidade e mortalidade maior que crianças não portadoras do vírus e por vezes uma qualidade de vida prejudicada ${ }^{28}$, cabendo então aos profissionais da saúde saber identificar e intervir nestas situações.

Os dados do presente estudo levaram à conclusão de que as crianças desta pesquisa demonstraram uma qualidade de vida influenciada pelos aspectos negativos presentes em suas vidas. Desta forma, é necessária uma equipe multidisciplinar para o tratamento e prevenção de comorbidades, inclusive as psicossociais.

Esta pesquisa contribuiu para um maior entendimento da infecção pelo vírus HIV em pacientes jovens e, ainda, como a infecção pode afetar o padrão de qualidade de vida dessas crianças. No entanto, deve-se ressaltar que os resultados obtidos, principalmente em relação aos domínios de qualidade de vida, não podem ser entendidos como definitivos e imutáveis, pois, devido ao seu caráter dinâmico, a epidemia do HIV/AIDS passa por diversas modificações com o decorrer do tempo, e a qualidade de vida das pessoas com o vírus, felizmente, só tende a melhorar.

\section{REFERÊNCIAS}

1. Rodrigues CS, Perreault M. A feminização do HIV/AIDS sob a perspectiva de mulheres infectadas: uma questão de gênero. In: Anais do 30 Seminário Internacional Enlaçando Sexualidades; 2013 maio 15-17; Salvador, Brasil. Salvador: UNEB; 2013.

2. Galvão MTG, Cunha GH, Freitas JG, Gir E, Reis RK. Sociodemographic, maternal and clinical conditions of children exposed to the human immunodeficiency vírus. Rev Rene. 2014;15(1):78-88. http://dx.doi. org/10.15253/2175-6783.2014000100011.

3. Abdo RF, Cunha RV. Qualidade de vida em crianças infectadas pelo HIV / AIDS. Bol Acad Paul Psicol. 2013;33(84):30-40.

4. Braz MM, Marcuzzo L, Ávila C. Efeitos de um programa de cinesioterapia sobre as condições de saúde de uma criança soropositiva. Cinergis. 2014;15(3):152-7.

5. Galvão MTG, Cunha GH, Rodrigues NLC, Gir E. Aspects of social interactions of HIV-positive children from the perspective of their caregivers. Rev Rene. 2013;14(1):262-71.

6. Brasil. Ministério da Saúde. Secretaria de Vigilância em Saúde. Programa Nacional de DST e AIDS. Boletim Epidemiológico DST/AIDS. Brasília (DF); 2012.

7. Friedrich L, Menegotto M, Magdaleno AM, Silva CLO. Transmissão vertical do HIV: uma revisão sobre o tema. Boletim Científico de Pediatria. 2016;5(3):81-86.

8. Joint United Nations Programme on HIV/AIDS. Get on the Fast Track: The life-cycle approach to HIV. Genebra: UNAIDS; 2016.

9. Rodrigues SLT, Vaz MJR, Barros SMO. Vertical transmission of HIV in the population treated at a reference center. Acta Paul Enferm. 2013;26(2):158-64. http://dx.doi.org/10.1590/S0103-21002013000200009.

10. Joint United Nations Programme on HIV/AIDS. AIDS by the numbers. Genebra: UNAIDS; 2016. 
11. Joint United Nations Programme on HIV/AIDS. Prevention Gap Report. Genebra: UNAIDS; 2016.

12. Brasil. Ministério da Saúde. Secretaria de Vigilância em Saúde. Programa Nacional de DST e AIDS. Boletim Epidemiológico DST/AIDS. Brasília; 2016.

13. Silva ACOS, Reis RK, Nogueira JA, Gir E. Qualidade de vida, características clínicas e adesão ao tratamento de pessoas vivendo com HIV/AIDS. Rev. Latino-Am. Enfermagem. 2014;22(6):994-1000. http://dx.doi. org/10.1590/0104-1169.3534.2508.

14. Organização Mundial de Saúde. Qualidade de Vida. Genebra: OMS; 1994.

15. Pinto DS, Vila VB, Vila RB, Marin HA, Almeida DR, Campos FMC. Estudo Bibliográfico da qualidade de vida dos portadores de HIV/AIDS. Rev Eletr Gestão \& Saúde. 2014;5(2):686-99.

16. Lima ICV, Pedrosa NL, Aguiar LFP, Galvão MTG. Demandas de cuidado domiciliar da criança nascida exposta ao HIV na ótica da teoria ambientalista. Rev Gaúcha Enferm. 2013;34(3):64-71. http://dx.doi.org/10.1590/ S1983-14472013000300008. PMid:24344586.

17. Motta MGC, Issi HB, Ribeiro AC, Botene DZA, Silva MC, Sengik SAMBS. Vivências da criança com HIV/AIDS. Cienc Cuid Saude. 2012;11(4):681-6.

18. Pereira AP, Petreça DR. Percepção e nível de qualidade de vida entre pré-escolares. R Bras Qual Vida. 2015;7(2):56-64.

19. Oliveira JF, Oliveira KF, Zago GP, Weffort VRS, Simões ALA. Qualidade de vida de crianças e adolescentes infectados pelo HIV. Cienc Cuid Saude. 2015;14(1):879-84. http://dx.doi.org/10.4025/cienccuidsaude. v14i1.19265.

20. Manificat S, Dazord A. Évaluation de la qualité de vie de l'enfant: Validation d'un questionnaire, premiers résultats. Neuropsychiatr Enfance Adolesc. 1997;45(3):106-14.

21. Assumpção FB Jr, Kuczynski E, Sprovieri MH, Aranha EMG. Escala de Avaliação de Qualidade de Vida (AUQEI - Autoquestionnaire Qualité de Vie Enfant Imagé) - Validade e confiabilidade de uma escala para qualidade de vida em crianças de 4 a 12 anos. Arq Neuropsiquiatr. 2000;58(1):119-27. http://dx.doi. org/10.1590/S0004-282X2000000100018. PMid:10770876.

22. Ferreira DC, Passos MRL, Rubini NPM, Knupp RRS, Curvelo JAR, Reis HLB, et al. Validation study of a scale of life quality evaluation in a group of pediatric patients infected by HIV. Cien Saude Colet. 2011;16(5):264352. http://dx.doi.org/10.1590/S1413-81232011000500034. PMid:21655738.

23. Buczynski AKC. Qualidade de vida relacionada à saúde oral em pacientes infantis infectados pelo HIV [dissertação]. Rio de Janeiro: Universidade Federal do Rio de Janeiro; 2007.

24. Bragheto ACM. Crianças portadoras do HIV/AIDS: desenvolvimento emocional e competência social [dissertação]. Ribeirão Preto: Escola de Enfermagem de Ribeirão Preto; 2008.

25. Ferreira JC. Qualidade de vida nas perspectivas de crianças e adolescentes portadores de HIV/AIDS [dissertação]. Goiás: Universidade Católica de Goiás; 2008.

26. Andrade ALDL, Alves CAJ, Medeiros MB, Galvão HC, Rosa MRD. Quality of life and oral health experience in HIV-infected and non-infected children. Rev Gaucha Odontol. 2012;60(3):343-8.

27. Nicastro E, Continisio GI, Storace C, Bruzzese E, Mango C, Liguoro I, et al. Family group psychotherapy to support the disclosure of HIV Status to children and adolescents. AIDS Patient Care STDS. 2013;27(6):3639. http://dx.doi.org/10.1089/apc.2012.0465. PMid:23691925.

28. Gomes GC, Pintanel AC, Strasburg AC, Xavier DM. Face singular do cuidado familiar à criança portadora do vírus HIV/AIDS. Acta Paul Enferm. 2012;25(5):749-54. http://dx.doi.org/10.1590/S010321002012000500016. 